\title{
Language, cognition, and the nature of modularity: Evidence from aphasia
}

\author{
Rosemary Varley ${ }^{\mathrm{a}}$ and Michael Siegal ${ }^{\mathrm{b}}$ \\ a Department of Human Communication Sciences, University of Sheffield, \\ Sheffield, UK S10 2TA; ${ }^{\mathrm{b}}$ Department of Psychology, Western Bank, \\ University of Sheffield, Sheffield, UK S10 2TP. R.A.Varley@sheffield.ac.uk \\ M.Siegal@sheffield.ac.uk
}

\begin{abstract}
We examine Carruthers's proposal that sentences in logical form serve to create flexibility within central system modularity, enabling the combination of information from different modalities. We discuss evidence from aphasia and the neurobiology of input-output systems. This work suggests that there exists considerable capacity for interdomain cognitive processing without language mediation. Other challenges for a logical form account are noted.
\end{abstract}

Peter Carruthers provides an elegant tour across the landscape of competing claims on the role of language in human cognition. This in itself is a complex undertaking, in view of the still limited evidence. For example, the evidence from aphasia only provides a window on the role of language in the mature cognitive system and says nothing as to how language might sculpt a distinctively human cognitive architecture. Hence a whole class of "diachronic" claims regarding the effects of language on cognition lie outside of the scope of the evidence from aphasia. Moreover, the evidence from aphasia studies of either associations or dissociations between language impairment and other cognitive functions should be interpreted with caution. Evidence of associations - for example, between aphasia and an inability to generate alternative strategies on a problem-solving task (Varley 2002; Varley et al. 2001) - falls far short of what is needed to draw causal inferences. Associations may result from naturally occurring brain lesions crossing the boundaries of the neural substrates of processing systems; the association is then an anatomical coincidence rather than a causal relationship in which language gives rise to cognition. Equally, dissociations between language impairment in aphasia and the sparing of cognitive performance in other domains such as space and number require careful consideration. In many studies, either the nature and extent of linguistic impairment are not clearly specified, or the abilities that are specified are such that the aphasic individuals have considerable residual language capacity still available to them that could support cognition. This capacity would be sufficient to generate simple "proto-language" - a language that is characterized by combinatorial properties but does not have the full features of a mature grammatical system (Bickerton 1981).

The proposal of massive central system modularity requires a mechanism that allows flexibility between domains of cognition. Although the notion of modularity is subject to different interpretations (e.g., Coltheart 1999; Fodor 1983), it is widely acknowledged that this must involve core characteristics such as domain specificity, informational encapsulation, and a proprietary operating code. However, flexibility and fluidity characterizes human cognition, particularly in higher-level reasoning and problem solving; hence the requirement for a cognitive lingua franca to combine the outputs of the modular subsystems. For Carruthers, the central code that permits the combination of outputs is logical form - the abstract, deep structural representations that underlie natural language sentences.

There are a number of possible reasons to reject the claim that natural language in the form of logical form sentences is the mech- 
anism for intermodular combination. For example, the nature of the brain architecture of input-output systems permits intermodal mixing at an early stage in information processing. Sensory-perceptual systems consist of batteries of micromodules that respond only to very specific inputs (e.g., the tonatopic organization of the primary auditory cortex). However, zones of association cortex, described as polymodal areas, surround primary sensory cortices. Association cortex receives inputs from a number of micromodules within a sensory domain such as vision or from different sensory domains. Tasks such as those of Hermer-Vazquez et al. (1999) that require the combination of visual object and geometric information are central to Carruthers's thesis on the relation between language and cognition. However, responses in such tasks could be achieved by a lower-level nonlinguistic perceptual mechanism. Evidence from language training studies that result in accurate spatial cognition could reflect a co-opted strategic resource that is sufficient to assist performance on a range of problem-solving tasks, including theory of mind (Siegal \& Varley 2002). There is debate about the extent of association cortex, with the suggestion that as perception is gradually fractionated into further micromodules, more of what is believed to be polymodal cortex may be reclassified as primary, sensory cortex (Kaas 1999). However, it is well established that the architecture of sensory-perceptual cortices permits the integration of information from different domains at an early stage in cognitive processing.

Another neurobiological example of interdomain information combination involves mirror neuron systems. These are cells in motor regions that fire when an action is observed, thus providing a mechanism for input-output linkage again at a low level of information processing (Rizzolatti et al. 2001). Both polymodal association cortex and mirror neuron systems show that the brain is equipped with mechanisms to permit combination of interdomain information at an early stage in information processing. These neural systems are intrinsic to input-output modules and are remote both anatomically and functionally from central cognitive systems.

There are other difficulties at both the theoretical and empirical levels for an account based on central modularity. For example, Carruthers's account suggests that the language faculty generates logical form (LF) sentences in response to inputs from a range of central modules. This is a problem because now a fundamental criterion for modularity appears to be violated in that the language production system is able to take as input a multiplicity of codes from a range domain-specific modules. The language faculty, or at least its output component, then becomes an omnipotent domain-general system, and Carruthers's attempt to develop the thesis of central system modularity results in a breakout of domain generality elsewhere in cognition.

We conclude by noting that an LF account poses a considerable challenge for experimental research. Imagine a set of results from a Hermer-Vazquez et al. type of task in which one subgroup of people with aphasia shows the ability to combine landmark and geometric information whereas others do not. In our laboratory, we have carried out a pilot study involving persons with severe aphasia in which the Hermer-Vazquez et al. room procedure is scaled down to a table-top box. In this task, rather than disorientating the patient, the box is spun. Our subjects were capable of utilizing both landmark and geometric cues in locating a hidden object, pointing to the sparing of their spatial cognition. They could find objects under conditions of no concurrent activity as well as in a simple lexical verbal shadowing condition where residual lexical capacity was engaged by the concurrent activity. As the aphasic participants had a profound impairment of their public language, a counter to these results could be that, at a deep and underlying level, the LF representation was intact. It is difficult to determine how the integrity or otherwise of LF representations could be established, given that evaluation of language processing at both implicit and explicit levels requires the capacity to process surface form. Similarities in aphasic impairments across input and output modalities may be seen to indicate a loss of central lan- guage competence. But this inference is an imprecise and indirect metric of the integrity of logical form. An alternative account might postulate parallel input-output impairments - resulting in disturbance of public language that would mimic a central competence deficit. Thus a crucial element in the evaluation of Carruthers's model must be whether it generates testable hypotheses. 